\title{
A Study on the Relationship of Glycaemic Status with Nature of Lesions in Acute Stroke Patients
}

\author{
Justin Haobam $^{1}$, Chingakham Arunkumar ${ }^{2}$, Konjengbam Ghanachandra Singh ${ }^{3}$ \\ ${ }^{1}$ PGT, ${ }^{2}$ Assistant Professor, ${ }^{3}$ Professor, \\ Department of General Medicine, Jawaharlal Nehru Institute of Medical Sciences (JNIMS), Porompat, Imphal, \\ Manipur, India.
}

Corresponding Author: Chingakham Arunkumar

\begin{abstract}
Background: Stroke in patients of Diabetes Mellitus is a common complication all over the world. The present study was done with the aim to assess the blood glucose level in acute stroke patients of Manipur and find out any correlation with types, size and prognosis of different glycaemic group.
\end{abstract}

Method: The study was a prospective crosssectional study of all patients who were admitted as acute stroke in Medicine ward, Jawaharlal Nehru Institute of Medical Sciences (JNIMS), Imphal, Manipur. Clinical parameter including History and clinical examination findings were recorded. CT scan of brain, blood glucose level, HbA1c were taken in all the patients.

Results: Of 109 patients of acute stroke patients included in the study, 34 were known cases of Diabetes, 10 were newly detected Diabetes cases summing up to a total of 64 cases of Diabetes, 44 of the cases had stress hyperglycaemia and 21 were euglycaemic. Maximum cases consisted of ischaemic strokes $(84.4 \%)$ and maximum number of haemorrhagic strokes falls under the known diabetes group. None of the euglycaemic patients had any large sized lesions, maximum of them $(89.5 \%)$ had small sized lesions. Most of the stress glycaemia patients had medium sized strokes while the known diabetes and newly detected diabetes groups had no case of small sized lesion with the maximum large sized lesions occurring in the known diabetes group (78.9\%). Clinical outcome was worst in poor glucose control group with $70.3 \%$ fatality in this group while maximum clinical improvement was seen in Non-Diabetics $(84.6 \%)$ consisting of euglycaemia and stress hyperglycaemia groups.
Conclusion: There was a good correlation with admission day hyperglycaemia with the size of the stroke lesion in all cases of stroke. The diabetes especially poor glucose control group is associated with increased size of the stroke lesion, severity and poor clinical outcome.

Keywords: Stroke, diabetes mellitus, stress hyperglycaemia, euglycaemia.

\section{INTRODUCTION}

A stroke is defined as the abrupt onset of neurological deficit due to a focal vascular cause the diagnosis of which is further augmented by various laboratory investigations particularly brain imaging. ${ }^{1}$ Strokes or Cerebrovascular accidents are one of the most dreaded conditions frequenting the emergency department of a hospital among adults in the world, accounting about half of all adult neurological diseases. Cerebrovascular accident includes ischaemic stroke, haemorrhagic stroke, and cerebrovascular anomalies such as intracranial aneurysm, AV malformation and cortical venous thrombosis.

$11.3 \%$ cases of stroke patients were found to be known Diabetics in a study conducted among admitted stroke patients, underlying the importance of Diabetes Mellitus as an important associated condition or risk factor for stroke ${ }^{2}$. In fact among the macrovascular complications due to diabetes, stroke is the most common one and causes significant morbidity and mortality among people with diabetes, thus identifying diabetes as an important risk 
factor in the genesis of stroke. ${ }^{3}$ There is also well established analysis of various studies implying the increased risk of stroke in diabetes and increased prevalence rate of stroke in diabetes as compared to several populations. ${ }^{4}$ The mortality rate of stroke in diabetics is twice that of the general population. ${ }^{5}$ The fact that glucose tolerance deteriorates with age, and that increased age is one of the most important risk factor for developing stroke has made the baseline blood glucose level of the individual especially the more aged ones an important risk factor for development of stroke. ${ }^{6}$ Blood glucose is also a significant independent predictor of death in cases of acute stroke, this has been shown by a multivariate study by Yadav et al. ${ }^{7}$

Long since Claude Bernard's observation of acutely elevated blood glucose levels in response to stress there has been studies documenting the presence of such states in stroke patients too. ${ }^{8}$ Aptly named stress hyperglycaemia which is a common immediate post stroke finding, is a state of acute stress responsive hyperglycaemia found in non-diabetics. This type of acutely developing hyperglycaemia found among non-diabetic patients has been shown to implicate a more severe neurological damage compared to those with normal baseline blood sugar levels on presentation. ${ }^{\text {? }}$

Hence, the present study has been designed to assess the glycaemic status of acute stroke patients, and study the clinical correlation of the glycaemic status of the patients including those with stress hyperglycaemia with the type and size of the stroke lesion and to determine whether admission day glycaemic status has any relation with the short term clinical outcome of such acute stroke patients.

\section{MATERIALS AND METHODS}

This study was a prospective crosssectional study of patients who were diagnosed as acute stroke cases. The study was carried out in the Department of Medicine, Jawaharlal Nehru Institute of
Medical Sciences, Imphal, Manipur. The present study was done during the period of one and half year starting from August 2018 till February 2020. 109 patients who were admitted in Medicine wards of JNIMS Hospital, Imphal East with acute stroke and within 72 hours after onset of symptoms irrespective of age, sex, religion and socioeconomic status were included in the study. Any pre-existing neurological disability from previous stroke or other diseases, cases that present with stroke like syndromes and stroke cases admitted after more than 72 hours from the onset of symptoms are excluded from the study. All cases underwent thorough clinical examination and CT scan of brain was performed in all the cases within 3 days of onset of symptoms to confirm the diagnosis and ascertain the type of stroke (ischaemic/haemorrhagic) and size of stroke (small, medium or large). The small-sized stroke was defined as $5 \mathrm{~mm}$ in diameter and not visible in more than two adjacent slices; large-sized stroke was defined as more than $10 \mathrm{~mm}$ in diameter or involving one complete vascular territory. The mediumsized was in between small and large.

The plasma venous glucose level was taken within 24 hours after admission for every patient. Glycosylated haemoglobin (HbA1C) was done to ascertain whether it was stress diabetes or newly diagnosed diabetes. Patients were divided into four groups: 1. Euglycaemic patients having random blood glucose (RBS) < 140mg/dl and glycosylated haemoglobin (HbA1C) $<6.5 \%$, 2. Patients with stress hyperglycaemia (with no history of diabetes) having RBS $>140 \mathrm{mg} / \mathrm{dl}$ and HbA1C <6.5\%, 3. Newly diagnosed diabetics (no history of diabetes) having RBS $>140 \mathrm{mg} / \mathrm{dl}$ and $\mathrm{HbA} 1 \mathrm{C}>6.5 \%$ and 4 . Known diabetics, diagnosis based on the criteria of American Diabetic association. Prior permission was taken from the ethical board JNIMS, Imphal before the study was conducted. Informed consent of the participants of the study was taken as per ethical committee guidelines. 


\section{STATISTICAL METHODS}

Data from all the patients selected in this study were used in the analysis. SPSS 20 version was used for statistical calculation.

\section{RESULTS}

Sex and age distribution: Out of total 109 patients who were included in this study, $74(67.9 \%)$ are males and $35(32.1 \%)$ are female. Only $1(0.9 \%)$ patient is in age group of upto 40 years, $12(11 \%)$ in the age group of 41 to 50 years, $59(54.1 \%)$ in 51 to 60 years, $28(25.7 \%)$ in 61 to 70 years, $8(7.3 \%)$ in 70 to 80 years and $1(0.9 \%)$ in 81 to 90 years group. The mean age of the patients in this study was $58 \pm 7.9$ years. Maximum number of patients belonged to the age group of 51-60 years.

Glycaemic status: In the study group, $34(31.2 \%)$ were known cases of Diabetes, while 10(9.2\%) were newly detected Diabetes cases and this summed up to a total of 44(40.4\%) cases of Diabetes, 44(40.4\%) of the cases had stress hyperglycaemia whereas only 21(19.2\%) were Euglycaemic.

Types of stroke in different glycaemic status groups: Out of 21 patients in euglycaemia group, 2 have medium and 19 small sized lesions on CT Brain, for 34 known diabetes, 15 have large and 19 medium sized lesions, out of 10 newly detected diabetes, 1 have large and 9 medium sized lesions and of 44 stress hyperglycaemia, 3 have large, 40 medium and 1 small sized lesions. In the euglycaemia group, $1(0.9 \%)$ has haemorrhagic stroke and 20(18.3\%) have ischaemic stroke, for known diabetes $11(10.1 \%)$ and $23(21.1 \%)$, in newly detected diabetes $0(0 \%)$ and $10(9.2 \%)$ and for stress hyperglycaemia 5(4.6\%) and $39(35.8 \%)$ respectively (Table No. 1 )

Table no.1: Types of stroke in different glycaemic status groups

\begin{tabular}{|l|c|c|c|}
\hline Glycaemic Status & Haemorrhagic stroke & Ischaemic Stroke & Total \\
\hline Euglycaemic & $1(0.9 \%)$ & $20(18.3 \%)$ & $21(19.2 \%)$ \\
\hline Known Diabetes & $11(10.1 \%)$ & $23(21.1 \%)$ & $34(31.2 \%)$ \\
\hline Newly Detected Diabetes & $0(0 \%)$ & $10(9.2 \%)$ & $10(9.2 \%)$ \\
\hline Stress Hyperglycaemia & $5(4.6 \%)$ & $39(35.8 \%)$ & $44(40.4 \%)$ \\
\hline Total & $17(15.6 \%)$ & $92(84.4 \%)$ & $109(100 \%)$ \\
\hline
\end{tabular}

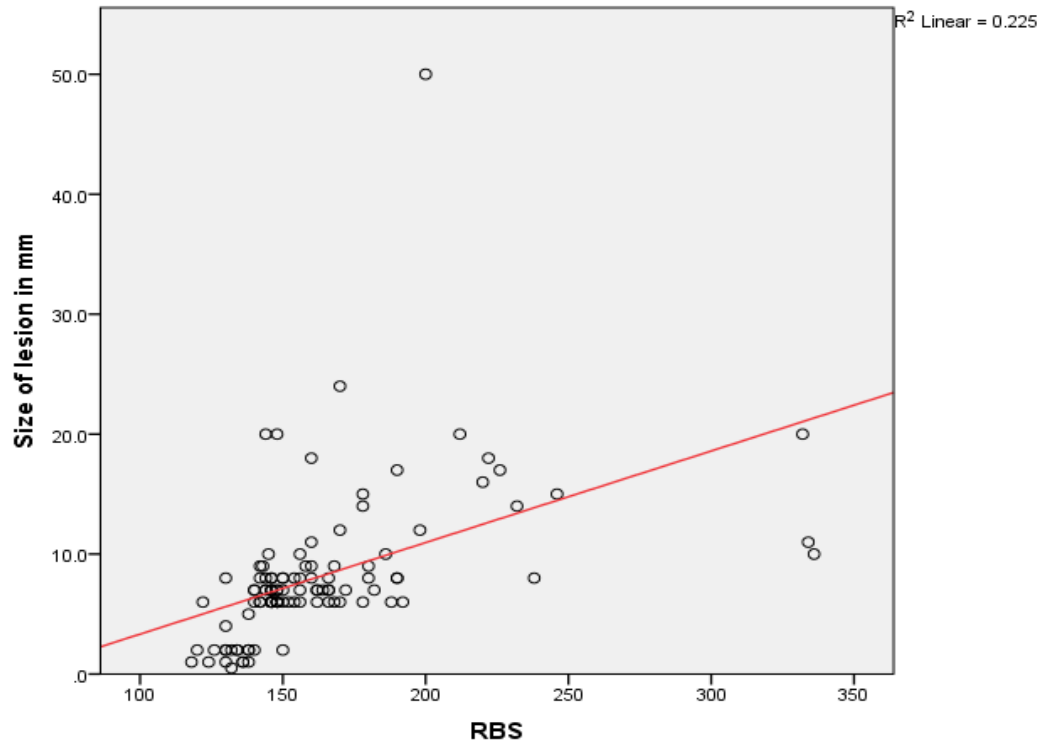

Graph no. 1: Correlation between admission RBS and size of the lesion

While the maximum cases consisted of ischaemic strokes $(84.4 \%)$ the maximum number of haemorrhagic stroke patients fall under the known diabetes group. None of the euglycaemic patients had any large sized lesions, with the maximum of them (89.5\%) 

patients.

having small sized lesions. Most of the stress glycaemia patients had medium sized strokes while the known diabetes and newly detected diabetes groups had no case of small sized lesion with the maximum large sized lesions occurring in the known diabetes group $(78.9 \%)$. This is significant as $\chi^{2}=110.59$ and $\mathrm{p}<0.001$ in this case. There is a positive correlation, $\mathrm{r}=0.475$ and $\mathrm{p}<0.001$ between the size of the stroke lesion and the admission day glucose implying that the size of the lesion increased as the glucose levels increased (Graph. No 1.).

Clinical outcome: In the study, clinical outcome of different glycaemic groups is shown in Table No. 2.

Table. No 2: Clinical outcomes in the different glycaemic status groups
\begin{tabular}{|l|c|l|l|c|}
\hline Glycaemic Status & Death & Improved & Referred & Total \\
\hline Euglycaemia & 0 & 21 & 0 & 21 \\
\hline Known diabetes & 17 & 8 & 9 & 34 \\
\hline Newly detected diabetes & 4 & 5 & 1 & 10 \\
\hline Stress hyperglycaemia & 6 & 34 & 4 & 44 \\
\hline Total & 27 & 68 & 14 & 109 \\
\hline
\end{tabular}

Maximum number of death and cases with further deterioration which involved referral to higher centres occurred among those with diabetes and stress hyperglycaemia whereas no death was reported in the Euglycaemia group with all of them eventually improving.

Different groups of degree of glucose control classified according to HbA1c levels (Table No 3) were also studied and Clinical outcome of different groups of glucose control are shown in Table No 4.

Table No.3: Classification of degree of glucose control by HbA1c levels

\begin{tabular}{|l|l|}
\hline Interpretation of HbA1C level & Degree of Glucose Control \\
\hline$>10 \%$ & Poor \\
\hline $9-10 \%$ & Fair \\
\hline $8-9 \%$ & Good \\
\hline $7-8 \%$ & Excellent \\
\hline $6-7 \%$ & Near-normal glycaemia \\
\hline$<6 \%$ & Non-diabetic \\
\hline
\end{tabular}

Table No. 4: Degree of glucose outcome and clinical outcome

\begin{tabular}{|l|l|l|l|l|}
\hline Degree of glucose control & Death & Improved & Referred & Total \\
\hline Excellent & 0 & 3 & 1 & 4 \\
\hline Fair & 1 & 3 & 1 & 5 \\
\hline Good & 1 & 0 & 1 & 2 \\
\hline Near normal glycaemia & 0 & 6 & 0 & 6 \\
\hline Non diabetic & 6 & 55 & 4 & 65 \\
\hline Poor & 19 & 1 & 7 & 27 \\
\hline Total & 27 & 68 & 14 & 109 \\
\hline
\end{tabular}

Clinical outcome was worst for those with poor glucose control with death occurring in $70.3 \%$ of this group while maximum number of patients with clinical improvement was seen in Non-Diabetics $(84.6 \%)$ which included patients from both the euglycaemia and stress hyperglycaemia groups.

\section{DISCUSSION}

The study was conducted at a tertiary care hospital in Imphal in 109 acute stroke patients who met the inclusion criteria. Most of the patients were in the age group of 51-60 years in this study with the mean age of $58 \pm 7.9$ years which is similar to the findings of Umpierrez GE et al ${ }^{9}$ where the mean age was $59 \pm 4$ years. There were 74 males and 35 females with a male preponderance cumulating to around $68 \%$ of the total cases which is comparable to the finding of Sharma et al ${ }^{10}$ and the findings of Weir et al implicating male sex as an important risk factor of stroke in patients presenting with hyperglycaemia. ${ }^{11}$

In this study the majority of the strokes were ischaemic and were mainly in the stress hyperglycaemic patients. There is also a high prevalence of known diabetics presenting with acute stroke $(31.2 \%)$ this is in contrast to the finding of Gracy et al and Keirs et al who found the prevalence of 
stroke in known diabetics to be $8.5 \%$ and $17 \%$ respectively. ${ }^{12,13}$ This higher prevalence may be due to lack of health awareness and life style, including the dietary habit of this geographical area compared to the majority of western country population of the other studies.

The study also showed that $9.2 \%$ of the patients presenting with acute strokes were newly diagnosed diabetics. Sharma et al reported as $16 \%$ and $12 \%$ in known diabetics and newly diagnosed diabetics respectively. ${ }^{10}$ These are in agreement with the observations in the series of Kiers et $\mathrm{al}^{13}$ and Sharma et $\mathrm{al}^{10}$, the prevalence of undiagnosed diabetes in acute stroke population varied from $6 \%$ to $42 \%$ (Riddle $\mathrm{MC}$ et al) in other studies. ${ }^{14} \mathrm{~A}$ significant population of this study amounting to a staggering $80.8 \%$ had above normal increased admission day glucose irrespective of their previous glycaemic status which is a notable finding.

Though the majority of the stroke was that of the Ischaemic variety across all glycaemia groups there is only one case of a Haemorrhagic stroke among the euglycaemia group amounting to only $0.9 \%$ of the study population while $15.6 \%$ of the study population who had Haemorrhagic stroke had admission day hyperglycaemia, indicating more number of haemorrhagic strokes among those patients with hyperglycaemia when compared to Euglycaemia patients.

The size of the stroke was measured with CT scan and was divided into three categories viz., small, medium and large, this categorisation is similar to the one done by Yadav et al. ${ }^{7}$ In our study $19(17.4 \%)$, $70(64.2 \%)$ and $20(18.4 \%)$ of the patients had large, medium and large sized lesions respectively whereas none of the euglycaemic patients had any large sized strokes the known diabetes group had the maximum number of large sized strokes $(13.8 \%)$.The percentages of small sized strokes in the euglycaemia, and stress hyperglycaemia groups were respectively $17.4 \%$ and $0.9 \%$ and the diabetes and newly detected diabetes group had no case of small sized lesions. The percentages of medium sized lesions in the euglycaemia, known diabetes, newly detected diabetes and stress hyperglycaemia groups were respectively $1.8 \%, 17.4 \%, 8.3 \%$ and $36.7 \%$ respectively. This was statistically significant as $\chi^{2}=$ 110.59 and $\mathrm{p}<0.001$. Thus, the size of the lesion increases as the status worsens for these patients and our study clearly shows a positive correlation $(\mathrm{r}=0.47$ and $\mathrm{p}<0.001)$ (Graph no.1) between admission day blood sugar level and size of the stroke lesion.

This is consistent with the findings in other studies who also reported increased size of stroke lesions in hyperglycaemia patients. ${ }^{13,15,16,17}$

This study revealed higher mortality with stress hyperglycaemia and diabetes, with maximum clinical improvement or recovery among the euglycaemia group which are consistent with other series as reported by some authors. ${ }^{9,12,18,19}$ A study published in the Journal of clinical endocrinology and metabolism, 2002 confirmed that patients with newly detected hyperglycaemia had a significant higher early mortality and a lower functional outcome than patients with a history of diabetes or normoglycemia which is consistent with the findings in our study. ${ }^{9}$ Gentile et al found admission day hyperglycaemia was an independent predictor of mortality in stroke patients even after disease severity was adequately addressed. ${ }^{19}$

The euglycaemia group had the best outcome among the various study groups with no mortality reported in this group. This finding is consistent with the findings of other studies of glycaemic status and prognosis that there was complete functional recovery in majority of the patients with normal glucose levels on admission.

Capes SE et al after the analysis of 32 similar studies implied that hyperglycaemic patients had threefold increased early mortality than euglycaemic patients. Admission day hyperglycaemia in 
case of ischaemic strokes was associated with three-fold increased 30 day mortality than eugylcaemics but the same pattern was not seen for cases of haemorrhagic stroke as admission hyperglycaemia was not associated with higher mortality in either diabetic or non-diabetic patients. ${ }^{17}$

This study also found the HbA1C levels corroborated well with the outcome of the patients with dead occurring in 19 of the patients whose HbA1c levels were more than $10 \%$ and were labelled as having 'poor' control where as those whose HbAlc levels were in between $6 \%$ to $8 \%$ falling in the groups labelled as having 'near normal glycaemia' and 'excellent' control had no reported death among them.

The present study clearly shows an increased early mortality rate and poor functional recovery in patients with diabetes and stress hyperglycaemia when compared to euglycaemia patients. Therefore, the need to confirm the improvement in these patients by normalizing blood sugar is imperative. Though some trails are now under way with the main objective of improvement of the outcome of stroke patients by normalizing the blood glucose with human recombinant insulin which showed that administration of insulin to patients with hyperglycaemia improves functional recovery and vital activity of mild to moderate ischemic stroke patients but the other potential clinical benefits of the insulin therapy are yet to be determined along with the clinically acceptable universal threshold for such an approach when treating acute stroke patients with hyperglycaemia. ${ }^{20,21}$

In the last decade, the incidence of ischaemic stroke and of related in-hospital mortality has reduced in persons with diabetes, however stroke incidence along with poor outcome and increasing severity is now seen among diabetic patients of advanced age partly due to the fact that the average diabetic patient's longevity in terms of their lifespan has increased tremendously compared to the previous decade. Women with diabetes, compared with men had a higher-in-hospital mortality risk. ${ }^{22}$

The preventive measures including good living habits, such as smoking cessation, limiting alcohol and avoiding high glucose and high fat diet, are very effective ways to prevent strokes. Good adherence to these measures helps in decreasing the morbidity and mortality in stroke patients with diabetes and improves the patient life quality. ${ }^{23}$ Thus, good preventive measures that takes into consideration all risk factors including good glycaemic control and effective treatment modality that adequately addresses the glycaemic status of the patient on presentation can help in prevention of stroke, limitation of its progression and aid in obtaining favourable outcomes including full functional recovery and reduction of mortality in acute stroke patients. ${ }^{20,21,24}$

Some limitations of this study were the exclusion of cases of stroke due to haematological disorders and intra cerebral pathology existing from before the acute stroke. The patients were not adequately followed up except for in hospital stay period, better correlation would have been possible had these patients been followed for a long term after discharge from the hospital. The present study did not address the question whether treatment of hyperglycaemia would have any effect on reducing the morbidity and mortality associated with stroke. The presence and effects of other confounding factors like hypertension, dyslipidaemia, cardiac arrhythmias etc. were also not considered.

\section{CONCLUSION}

Hyperglycaemia was a common finding in all cases of acute stroke patients including those with no previous history of diabetes. There was a good correlation with admission day hyperglycaemia with the size of the stroke lesion in all cases of stroke. The stress hyperglycaemia and diabetes groups had the maximum number of medium and large sized strokes while there was no case of large sized strokes among 
the euglycaemia patients. Maximum number of small sized strokes occurred in the euglycaemia group. The majority of the strokes were ischaemic; highest number of haemorrhagic cases was seen in patients with increased blood glucose levels on admission. Functional outcome was poor for patients with increased glucose levels when compared to those presenting with normal glucose levels. Mortality was maximum in those patients with diabetes irrespective of the size of the lesion. High HbA1c was also associated with increased mortality irrespective of the admission day hyperglycaemia or size of lesion.

The findings of this study shows that most of the acute stroke patients have elevated blood glucose levels irrespective of diabetes background and is associated with increased size of the stroke lesion, severity and poor clinical outcome. A reduction of blood glucose level to near normal levels is recommended to reduce the stroke progression and size of the stroke lesion and eventually improving the outcome of acute stroke events while recognising the deleterious effects of hypoglycaemia in these patients.

\section{Funding: None \\ Conflict of interest: None declared}

\section{REFERENCES}

1. Smith WS, English JD, Johnston SC. Cerebrovascular disease. In: Harrison's principles of internal medicine. 18th edn. New York: McGraw Hill 2011:3270-3249.

2. Frederic MW. Cerebrovascular disease. In: Conn HLJR, Horwitzo, eds. Cardiac and vascular disease. Philadelphia: Lea \& Febiger 1971:1473-1499.

3. Joshi SR, Shah SN. Rising global burden of diabetes. The Asian J Diabetology 1996; 1(3):13-15.

4. Wolf PA, Cobb JL, D' Agostine. Epidemiology of stroke. In: Barnett HJM, Mohr JP, eds. Stroke. 2nd edn. Edinburg: Churchill Livingstone 1992:3-27.

5. Houseley E. Definition of risk factors in stroke. In: Gillingam FJ, Mawdsley C, eds. Stroke. New York: Churchill Livingstone 1976:251-260.
6. Davidson MB. The effect of ageing on carbohydrates metabolism. A review of the English literature and a practical approach to the diagnostic of diabetes mellitus in the elderly. Metabolism 1979;28(6):688-705.

7. Yadav K, Chaudhary R. Clinical profile and the outcome of stroke in relation to the glycaemic status of patients. JIMA Vol 103 No.3 Mar 2004;138-40

8. Bernard C. Lecons sur de diabete at la glycogenase animale. Paris, Baillaire; 1877.

9. Umpierrez GE, Isaacs SD, Bazargan N, You $X$, Thaler LM, Kitabchi AE. Hyperglycemia: an independent marker of in-hospital mortality in patients with undiagnosed diabetes. J Clin Endocrinol Metab. 2002 Mar;87(3):978-82

10. Sharma AK, Mahrotra TN, Goel VK, et al. Clinical, profile of stroke in relation to glycaemic status of patients. J Assoc Physicians India 1996;44(1):19-21.

11. Weir CJ, Murray GD, Dyker AG, et al. Is hyperglycaemia an independent predictor of poor outcome after acute stroke? Results of long term follow up study.BMJ 1997; 314(7090):1303-1306.

12. Gracy CS, French JM, Castlidge NEF, et al. Increasing, diabetes mellitus and recovery from stroke. Post graduate Medical Journal 1989;65(768):720-724.

13. Kiers L, Davis SM, Larkins R, et al. Stroke topography and outcome in relation to hyperglycaemia and diabetes. J Neurol Neurosung Psychiatry 1992;55(4):263-270.

14. Riddle MC, Hart J. Hyperglycaemia, recognized and unrecognized as a risk factor for stroke and transient ischaemic attacks. Stroke 1982;13(3):356-359.

15. Prado R, Ginsberg MD, Dietrich WD et al. Hyperglycemia increases infarct size in collaterally perfused but not end-arterial vascular territories. J Cereb Blood Flow Metab 1988;8:186-92.

16. Gisselsson L, Smith ML, Siesjo BK. Hyperglycemia and focal brain ischemia. J Cereb Blood Flow Metab 1999;19:288-97.

17. Capes SE, Hunt D, Malmberg K et al. Stress hyperglycemia and prognosis of stroke in non diabetic and diabetic patients: a systematic overview. Stroke 2001;32:242632.

18. Candelise L, Landi G, Boccardi E, et al Prognostic Significance of Hyperglycemia in Acute Stroke, Arch Neurol. 1985;42(7): 661-663. 

patients.

19. Gentile, N.T., Seftchick, M.W., Huynh, T., Kruus, L.K. and Gaughan, J. (2006), Decreased Mortality by Normalizing Blood Glucose after Acute Ischemic Stroke. Academic Emergency Medicine, 13: 174180.

20. Bruno A, Kent TA, Coull BM, Shankar RR, Saha C, Becker KJ, et al. Treatment of hyperglycemia in ischemic stroke (THIS): a randomized pilot trial. Stroke. 2008 Feb. 39(2):384-9.

21. Bellolio MF, Gilmore RM, Stead LG. Insulin for glycaemic control in acute ischaemic stroke. Cochrane Database Syst Rev. 2011 Sep 7;1:26-32.

22. Bushnell C, McCullough LD, Awad IA, Chireau MV, Fedder WN, Furie KL, et al. Guidelines for the Prevention of Stroke in Women: A Statement for Healthcare Professionals From the American Heart
Association/American Stroke Association. Stroke.2014 Feb.45:1545-88.

23. Goldstein LB, Bushnell CD, Adams RJ, Appel LJ, Braun LT, Chaturvedi S, et al. Guidelines for the primary prevention of stroke: a guideline for healthcare professionals from the American Heart Association/American Stroke Association. Stroke.2011 Feb. 42(2):517-84.

24. Adams HP Jr, del Zoppo G, Alberts MJ, Bhatt DL, Brass L, Furlan A, et al. Guidelines for the early management of adults with ischemic stroke. Stroke. 2007 May. 38(5):1655-711.

How to cite this article: Haobam J, Arunkumar C, Singh KG. A study on the relationship of glycaemic status with nature of lesions in acute stroke patients. Gal Int J Health Sci Res. 2021; 6(1): 13-20. 